\title{
Extending the Applicability and Convergence Domain of a Fifth-Order Iterative Scheme under Hölder Continuous Derivative in Banach Spaces
}

\author{
Debasis Sharma $^{1 *(\mathbb{D}}$, Sanjaya Kumar Parhi ${ }^{2}$, Shanta Kumari Sunanda ${ }^{1}$ \\ ${ }^{1}$ Department of Mathematics, IIIT Bhubaneswar, Odisha, India \\ ${ }^{2}$ Department of Mathematics, Fakir Mohan University, Odisha, India \\ E-mail: iiit.debasis@gmail.com
}

Received: 26 May 2021; Revised: 16 August 2021; Accepted: 24 August 2021

\begin{abstract}
The most significant contribution made by this study is that the applicability and convergence domain of a fifth-order convergent nonlinear equation solver is extended. We use Hölder condition on the first Fréchet derivative to study the local analysis, and this expands the applicability of the formula for such problems where the earlier study based on Lipschitz constants cannot be used. This study generalizes the local analysis based on Lipschitz constants. Also, we avoid the use of the extra assumption on boundedness of the first derivative of the involved operator. Finally, numerical experiments ensure that our analysis expands the utility of the considered scheme. In addition, the proposed technique produces a larger convergence domain, in comparison to the earlier study, without using any extra conditions.
\end{abstract}

Keywords: iterative schemes, Banach space, local convergence, Hölder continuity condition

MSC: 47H99, 49M15, 65J15, 65G99

\section{Introduction}

Let $\mathcal{X}$ and $\mathcal{Y}$ be Banach spaces. $\Omega \subseteq \mathcal{X}$ is a non-empty, open and convex set. Suppose $\mathcal{Q}: \Omega \subseteq \mathcal{X} \rightarrow \mathcal{Y}$ is a nonlinear operator, which is differentiable according to Fréchet. The primary goal of this research is to broaden the scope of the application and convergence domain of a fifth-order iterative scheme for solving nonlinear operator equations in the form

$$
\mathcal{Q}(s)=0
$$

Applications of these equations are found in many fields such as Engineering, Optimization, Economics, Chemistry, Biology, etc. For example, problems in radiative transfer theory, static dynamical equations, Kinetic theory of gases, and other topics can be solved using nonlinear equations of the form (1). For these equations, mostly iterative schemes (see books [1-6], see research papers [7-18]) are used. A fundamental and widely used scheme for addressing (1) is Newton's method, whose iteration procedure is given by

Copyright (C2021 Debasis Sharma, et al

DOI: https://doi.org/10.37256/cm.242021962

This is an open-access article distributed under a CC BY license

(Creative Commons Attribution 4.0 International License)

https://creativecommons.org/licenses/by/4.0/ 


$$
s_{k+1}=s_{k}-\left[\mathcal{Q}^{\prime}\left(s_{k}\right)\right]^{-1} \mathcal{Q}\left(s_{k}\right), k \geq 0 .
$$

On the other hand, finding convergence radius as well as constructing a technique to expand the domain of convergence are two important matters in the study of iterative schemes. Convergence domain of an iterative scheme is required to determine such initial points whose corresponding sequences converge to the solution. "The analysis of local convergence of an iterative scheme uses the information around a solution and provides the convergence radii" [1]. The local results for Chebyshev and Halley type procedures are provided in [19-21]. Also, important findings on the local analysis of fourth, fifth, and sixth order schemes are presented in [22-27]. More results on the local analysis of iterative procedures have been established in the literature [28-35]. In this paper, we expand the convergence domain as well as the applicability of a fifth-order scheme using Hölder condition on $\mathcal{Q}^{\prime}$ only.

The local convergence of the following fifth-order convergent scheme is suggested in [22] by Argyros and George.

$$
\begin{aligned}
t_{k} & =s_{k}-\left[\mathcal{Q}^{\prime}\left(s_{k}\right)\right]^{-1} \mathcal{Q}\left(s_{k}\right), \\
u_{k} & =t_{k}-\left[\mathcal{Q}^{\prime}\left(s_{k}\right)\right]^{-1}\left(\mathcal{Q}^{\prime}\left(s_{k}\right)-\mathcal{Q}^{\prime}\left(t_{k}\right)\right)\left(\mathcal{Q}^{\prime}\left(s_{k}\right)+\mathcal{Q}^{\prime}\left(t_{k}\right)\right)^{-1} \mathcal{Q}\left(s_{k}\right), \\
s_{k+1} & =u_{k}-\left(\mathcal{Q}^{\prime}\left(s_{k}\right)+\mathcal{Q}^{\prime}\left(t_{k}\right)\right)^{-1}\left(3 \mathcal{Q}^{\prime}\left(s_{k}\right)-\mathcal{Q}^{\prime}\left(t_{k}\right)\right)\left[\mathcal{Q}^{\prime}\left(s_{k}\right)\right]^{-1} \mathcal{Q}\left(u_{k}\right), k=0,1,2, \ldots
\end{aligned}
$$

They proved the convergence analysis of this scheme by applying the Lipschitz continuity of $\mathcal{Q}^{\prime}$, that is,

$$
\left\|\mathcal{Q}^{\prime}\left(s^{*}\right)^{-1}\left(\mathcal{Q}(s)-\mathcal{Q}\left(s^{*}\right)\right)\right\| \leq l_{0}\left\|s-s^{*}\right\|, \forall s \in \Omega
$$

and

$$
\left\|\mathcal{Q}^{\prime}\left(s^{*}\right)^{-1}(\mathcal{Q}(s)-\mathcal{Q}(t))\right\| \leq l\|s-t\|, \quad \forall s, t \in \Omega .
$$

However, there are many instances where the technique based on Lipschitz conditions fails. As an illustration, we take the following integral equation given in [27].

$$
\mathcal{Q}(s)(w)=s(w)-3 \int_{0}^{1} G_{1}(w, y) s(y)^{\frac{5}{4}} d y,
$$

where $s(w) \in C[0,1]$ and $G_{1}(x, y)$ is Green's function defined on $[0,1] \times[0,1]$ by

$$
G_{1}(w, y)=\left\{\begin{array}{ll}
(1-w) y, & \text { if } y \leq w \\
w(1-y), & \text { if } w \leq y
\end{array} .\right.
$$

Then,

$$
\left\|\mathcal{Q}^{\prime}(s)-\mathcal{Q}^{\prime}(t)\right\| \leq \frac{15}{32}\|s-t\|^{\frac{1}{4}} .
$$

It is noteworthy that $\mathcal{Q}^{\prime}$ is not Lipschitz continuous. Hence, the existing work [22] cannot be implemented to address the above problem. However, $\mathcal{Q}^{\prime}$ belongs to the Hölder continuity class. So, we establish the local convergence of the iterative scheme (3) by employing Hölder continuity of $\mathcal{Q}^{\prime}$.

In addition, the benefits of our analysis are: this study generalizes the local analysis based on Lipschitz constants given in [22] and expands the applicability of the formula (3). We avoid the use of the extra condition based on the 
boundedness of $\mathcal{Q}^{\prime}$. Also, we produce larger radii of convergence balls in comparison to the earlier study [22]. The most important point is that the proposed technique provides these advantages without using any extra conditions.

This manuscript is outlined as follows: Section 1 contains the introduction part. In Section 2, the analysis of local convergence of the iterative scheme (3) is described. Section 3 offers numerical applications of the proposed analytical results. The conclusion is provided in the final section.

\section{Local convergence analysis}

In order to expand the applicability and the convergence domain of the scheme (3), we establish the local analysis with the help of some real parameters and functions. Let $B(c, \gamma)$ and $\bar{B}(c, \gamma)$ denote open and closed balls, respectively, in $\mathcal{X}$ for $c \in \mathcal{X}$ and $\gamma>0 . B L(\mathcal{X}, \mathcal{Y})$ stands for the set of all linear and bounded operators from $\mathcal{X}$ to $\mathcal{Y}$. Let $q \in(0,1]$. Considering two positive parameters $w_{0}$ and $w_{1}$ with $w_{0} \leq w_{1}$, we define $\mathcal{H}_{1}$ on $\left[0,\left(\frac{1}{w_{0}}\right)^{\frac{1}{q}}\right]$ by

$$
\mathcal{H}_{1}(x)=\frac{w_{1} x^{q}}{(q+1)\left(1-w_{0} x^{q}\right)}
$$

and the parameter

$$
\Delta_{1}=\left(\frac{(q+1)}{(q+1) w_{0}+w_{1}}\right)^{\frac{1}{q}}<\left(\frac{1}{w_{0}}\right)^{\frac{1}{q}}
$$

Observe that $\mathcal{H}_{1}\left(\Delta_{1}\right)=1$. Again, we define functions $\mathcal{H}_{2}$ and $\mathcal{A}_{2}$ on $\left[0,\left(\frac{1}{w_{0}}\right)^{\frac{1}{q}}\right)$ by

$$
\mathcal{H}_{2}(x)=\frac{w_{0}}{2}\left(\mathcal{H}_{1}(x)^{q}+1\right) x^{q}
$$

and

$$
\mathcal{A}_{2}(x)=\mathcal{H}_{2}(x)-1
$$

Now, $\mathcal{A}_{2}(0)=-1<0$ and $\lim \mathcal{A}_{2}(x)=+\infty$. We apply intermediate value theorem (IVT) to show the existence $x \rightarrow\left(\left(\frac{1}{w_{0}}\right)^{\frac{1}{q}}\right)^{-}$

of the zeros of the function $\mathcal{A}_{2}(x)$ in the interval $\left(0,\left(\frac{1}{w_{0}}\right)^{\frac{1}{q}}\right)$. Let us denote the smallest zero of $\mathcal{A}_{2}(x)$ in $\left(0,\left(\frac{1}{w_{0}}\right)^{\frac{1}{q}}\right)$ by $\Delta_{2}$. Again, we define $\mathcal{H}_{3}$ and $\mathcal{A}_{3}$ on $\left[0, \Delta_{2}\right)$ by

$$
\mathcal{H}_{3}(x)=\mathcal{H}_{1}(x)+\frac{w_{0}\left(\mathcal{H}_{1}(x)^{q} x^{q}+x^{q}\right)\left(1+x^{q}\right)}{\left(1-w_{0} x^{q}\right)\left(2\left(1-\mathcal{H}_{2}(x)\right)\right)}
$$

and

$$
\mathcal{A}_{3}(x)=\mathcal{H}_{3}(x)-1
$$

Now, $\mathcal{A}_{3}(0)=-1<0$ and $\lim _{x \rightarrow \Delta_{2}^{-}} \mathcal{A}_{3}(x)=+\infty$. So, application of IVT gives us the smallest zero $\Delta_{3}$ of $\mathcal{A}_{3}(x)$ in the 
interval $\left(0, \Delta_{2}\right)$. Lastly, we introduce $\mathcal{H}_{4}$ and $\mathcal{A}_{4}$ on $\left[0, \Delta_{2}\right)$ by

$$
\mathcal{H}_{4}(x)=\mathcal{H}_{3}(x)+\frac{\left[w_{0}\left(\mathcal{H}_{1}(x)^{q} x^{q}+3 x^{q}\right)+2\right]\left(1+x^{q}\right) \mathcal{H}_{3}(x)}{\left(1-w_{0} x^{q}\right)\left(2\left(1-\mathcal{H}_{2}(x)\right)\right)}
$$

and

$$
\mathcal{A}_{4}(x)=\mathcal{H}_{4}(x)-1
$$
choose

Now, $\mathcal{A}_{4}(0)=-1<0$ and $\lim _{x \rightarrow \Delta_{2}^{-}} \mathcal{A}_{4}(x)=+\infty$. Then the minimal zero $\Delta_{4}$ of $\mathcal{A}_{4}(x)$ exists in $\left(0, \Delta_{2}\right)$ due to IVT. We

$$
R=\min \left\{\Delta_{1}, \Delta_{3}, \Delta_{4}\right\}
$$

to confirm the followings.

$$
\begin{aligned}
& 0 \leq \mathcal{H}_{1}(x)<1, \\
& 0 \leq \mathcal{H}_{2}(x)<1, \\
& 0 \leq \mathcal{H}_{3}(x)<1
\end{aligned}
$$

and

$$
0 \leq \mathcal{H}_{4}(x)<1
$$

for each $x \in[0, R)$. Also, we consider the following assumptions to prove the main convergence result. Let $\mathcal{Q}: \Omega \subseteq \mathcal{X}$ $\rightarrow \mathcal{Y}$ be a Fréchet derivable operator such that

$$
\begin{gathered}
\mathcal{Q}\left(s^{*}\right)=0, \mathcal{Q}^{\prime}\left(s^{*}\right)^{-1} \in B L(\mathcal{Y}, \mathcal{X}), \\
\left\|\mathcal{Q}^{\prime}\left(s^{*}\right)^{-1}\left(\mathcal{Q}^{\prime}(s)-\mathcal{Q}^{\prime}\left(s^{*}\right)\right)\right\| \leq w_{0}\left\|s-s^{*}\right\|^{q}, \forall s \in \Omega
\end{gathered}
$$

and

$$
\left\|\mathcal{Q}^{\prime}\left(s^{*}\right)^{-1}\left(\mathcal{Q}^{\prime}(s)-\mathcal{Q}^{\prime}(t)\right)\right\| \leq w_{1}\|s-t\|^{q}, \forall s, t \in \Omega_{0},
$$

where $\Omega_{0}=B\left(s^{*},\left(\frac{1}{w_{0}}\right)^{\frac{1}{q}}\right) \cap \Omega$.

Many authors $[22,28]$ use an extra assumption

$$
\left\|\mathcal{Q}^{\prime}\left(s^{*}\right)^{-1} \mathcal{Q}^{\prime}(s)\right\| \leq M
$$

We remove this additional condition using the following results.

Lemma 1 If $\mathcal{Q}$ obeys (15) and $\bar{B}\left(s^{*}, R\right) \subseteq \Omega$, then $\forall s \in B\left(s^{*}, R\right)$, we get 


$$
\left\|\mathcal{Q}^{\prime}\left(s^{*}\right)^{-1} \mathcal{Q}^{\prime}(s)\right\| \leq 1+w_{0}\left\|s-s^{*}\right\|^{q}
$$

and

$$
\left\|\mathcal{Q}^{\prime}\left(s^{*}\right)^{-1} \mathcal{Q}(s)\right\| \leq\left(1+w_{0}\left\|s-s^{*}\right\|^{q}\right)\left\|s-s^{*}\right\| .
$$

Proof. Applying (15), we get

$$
\left\|\mathcal{Q}^{\prime}\left(s^{*}\right)^{-1} \mathcal{Q}^{\prime}(s)\right\| \leq 1+\left\|\mathcal{Q}^{\prime}\left(s^{*}\right)^{-1}\left(\mathcal{Q}^{\prime}(s)-\mathcal{Q}^{\prime}\left(s^{*}\right)\right)\right\| \leq 1+w_{0}\left\|s-s^{*}\right\|^{q}
$$

For $\xi \in[0,1]$

$$
\left\|s^{*}+\xi\left(s-s^{*}\right)-s^{*}\right\|=\xi\left\|s-s^{*}\right\|<R \text {. }
$$

So, $s^{*}+\xi\left(s-s^{*}\right) \in B\left(s^{*}, R\right)$ and

$$
\left\|\mathcal{Q}^{\prime}\left(s^{*}\right)^{-1} \mathcal{Q}^{\prime}\left(s^{*}+\xi\left(s-s^{*}\right)\right)\right\| \leq 1+w_{0} \xi\left\|s-s^{*}\right\|^{q} \leq 1+w_{0}\left\|s-s^{*}\right\|^{q} .
$$

We apply the mean value theorem and (20) to find

$$
\begin{aligned}
\left\|\mathcal{Q}^{\prime}\left(s^{*}\right)^{-1} \mathcal{Q}(s)\right\| & =\left\|\mathcal{Q}^{\prime}\left(s^{*}\right)^{-1}\left(\mathcal{Q}(s)-\mathcal{Q}\left(s^{*}\right)\right)\right\| \\
& \leq\left\|\mathcal{Q}^{\prime}\left(s^{*}\right)^{-1} \mathcal{Q}^{\prime}\left(s^{*}+\xi\left(s-s^{*}\right)\right)\left(s-s^{*}\right)\right\| \\
& \leq\left(1+w_{0}\left\|s-s^{*}\right\|^{q}\right)\left\|s-s^{*}\right\| .
\end{aligned}
$$

Theorem 1 Let $s^{*} \in \Omega$ and the conditions (14)-(16) hold true and

$$
\bar{B}\left(s^{*}, R\right) \subseteq \Omega,
$$

where $R$ is given in (9). Then, for any starting point $s_{0} \in B\left(s^{*}, R\right)$ the iterative formula (3) produces the well defined sequence $\left\{s_{k}\right\}$ such that $\left\{s_{k}\right\}_{k \geq 0} \in B\left(s^{*}, R\right)$ and $\lim _{k \rightarrow \infty} s_{k}=s^{*}$. Also, the following items hold true for all $k \geq 0$

$$
\begin{aligned}
& \left\|t_{k}-s^{*}\right\| \leq \mathcal{H}_{1}\left(\left\|s_{k}-s^{*}\right\|\right)\left\|s_{k}-s^{*}\right\|<\left\|s_{k}-s^{*}\right\|<R, \\
& \left\|u_{k}-s^{*}\right\| \leq \mathcal{H}_{3}\left(\left\|s_{k}-s^{*}\right\|\right)\left\|s_{k}-s^{*}\right\|<\left\|s_{k}-s^{*}\right\|<R
\end{aligned}
$$

and

$$
\left\|s_{k+1}-s^{*}\right\| \leq \mathcal{H}_{4}\left(\left\|s_{k}-s^{*}\right\|\right)\left\|s_{k}-s^{*}\right\|<\left\|s_{k}-s^{*}\right\|<R
$$

where the real functions $\mathcal{H}_{1}, \mathcal{H}_{3}$ and $\mathcal{H}_{4}$ are introduced in (5), (7) and (8) respectively. For $\Delta \in\left[R,\left(\frac{q+1}{w_{0}}\right)^{\frac{1}{q}}\right), s^{*}$ uniquely solves the equation $\mathcal{Q}(s)=0$ in $\bar{B}\left(s^{*}, \Delta\right) \cap \Omega$.

Proof. Since $s_{0} \in B\left(s^{*}, R\right)$, we obtain 


$$
\left\|\mathcal{Q}^{\prime}\left(s^{*}\right)^{-1}\left(\mathcal{Q}^{\prime}\left(s_{0}\right)-\mathcal{Q}^{\prime}\left(s^{*}\right)\right)\right\| \leq w_{0}\left\|s_{0}-s^{*}\right\|^{q}<w_{0} R^{q}<1
$$

using (9) and (15). Now, Banach lemma $[1,5,6]$ guarantees that $\mathcal{Q}^{\prime}\left(s_{0}\right)^{-1} \in B L(\mathcal{Y}, \mathcal{X})$ and

$$
\left\|\mathcal{Q}^{\prime}\left(s_{0}\right)^{-1} \mathcal{Q}^{\prime}\left(s^{*}\right)\right\| \leq \frac{1}{1-w_{0}\left\|s_{0}-s^{*}\right\|^{q}}<\frac{1}{1-w_{0} R^{q}} .
$$

Therefore, $t_{0}$ is well defined. Again, we get from (3) that

$$
\begin{aligned}
t_{0}-s^{*} & =s_{0}-s^{*}-\mathcal{Q}^{\prime}\left(s_{0}\right)^{-1} \mathcal{Q}\left(s_{0}\right) \\
& =-\left[\mathcal{Q}^{\prime}\left(s_{0}\right)^{-1} \mathcal{Q}^{\prime}\left(s^{*}\right)\right]\left[\int_{0}^{1} \mathcal{Q}^{\prime}\left(s^{*}\right)^{-1}\left(\mathcal{Q}^{\prime}\left(s^{*}+\xi\left(s_{0}-s^{*}\right)\right)-\mathcal{Q}^{\prime}\left(s_{0}\right)\right)\left(s_{0}-s^{*}\right) d \xi\right] .
\end{aligned}
$$

Using (5), (9), (10), (26) and (27), we get

$$
\begin{aligned}
\left\|t_{0}-s^{*}\right\| & \leq\left[\left\|\mathcal{Q}^{\prime}\left(s_{0}\right)^{-1} \mathcal{Q}^{\prime}\left(s^{*}\right)\right\|\right]\left[\left\|\int_{0}^{1} \mathcal{Q}^{\prime}\left(s^{*}\right)^{-1}\left(\mathcal{Q}^{\prime}\left(s^{*}+\xi\left(s_{0}-s^{*}\right)\right)-\mathcal{Q}^{\prime}\left(s_{0}\right)\right)\left(s_{0}-s^{*}\right) d \xi\right\|\right] \\
& \leq \frac{w_{1}\left\|s_{0}-s^{*}\right\|^{q}}{(q+1)\left(1-w_{0}\left\|s_{0}-s^{*}\right\|^{q}\right)}\left\|s_{0}-s^{*}\right\| \\
& =\mathcal{H}_{1}\left(\left\|s_{0}-s^{*}\right\|\right)\left\|s_{0}-s^{*}\right\| \leq\left\|s_{0}-s^{*}\right\|<R .
\end{aligned}
$$

So, (22) is true for $k=0$. Now, we claim that $\left[\mathcal{Q}^{\prime}\left(s_{0}\right)+\mathcal{Q}^{\prime}\left(t_{0}\right)\right]^{-1} \in B L(\mathcal{Y}, \mathcal{X})$. The equations (6), (9), (11), (15) and (28) are used to obtain

$$
\begin{aligned}
& \left\|\left(2 \mathcal{Q}^{\prime}\left(s^{*}\right)\right)^{-1}\left[\mathcal{Q}^{\prime}\left(s_{0}\right)+\mathcal{Q}^{\prime}\left(t_{0}\right)-2 \mathcal{Q}^{\prime}\left(s^{*}\right)\right]\right\| \\
& \leq \frac{1}{2}\left[\left\|\mathcal{Q}^{\prime}\left(s^{*}\right)^{-1}\left(\mathcal{Q}^{\prime}\left(s_{0}\right)-\mathcal{Q}^{\prime}\left(s^{*}\right)\right)\right\|+\left\|\mathcal{Q}^{\prime}\left(s^{*}\right)^{-1}\left(\mathcal{Q}^{\prime}\left(t_{0}\right)-\mathcal{Q}^{\prime}\left(s^{*}\right)\right)\right\|\right] \\
& \leq \frac{w_{0}}{2}\left[\left\|s_{0}-s^{*}\right\|^{q}+\left\|t_{0}-s^{*}\right\|^{q}\right] \\
& \leq \frac{w_{0}}{2}\left[\left\|s_{0}-s^{*}\right\|^{q}+\mathcal{H}_{1}\left(\left\|s_{0}-s^{*}\right\|\right)^{q}\left\|s_{0}-s^{*}\right\|^{q}\right] \\
& =\frac{w_{0}}{2}\left[\mathcal{H}_{1}\left(\left\|s_{0}-s^{*}\right\|\right)^{q}+1\right]\left\|s_{0}-s^{*}\right\|^{q} \\
& =\mathcal{H}_{2}\left(\left\|s_{0}-s^{*}\right\|\right)<\mathcal{H}_{2}(R)<1 .
\end{aligned}
$$

Now, we obtain $\left[\mathcal{Q}^{\prime}\left(s_{0}\right)+\mathcal{Q}^{\prime}\left(t_{0}\right)\right]^{-1} \in B L(\mathcal{Y}, \mathcal{X})$ and 


$$
\left\|\left[\mathcal{Q}^{\prime}\left(s_{0}\right)+\mathcal{Q}^{\prime}\left(t_{0}\right)\right]^{-1} \mathcal{Q}^{\prime}\left(s^{*}\right)\right\| \leq \frac{1}{2\left(1-\mathcal{H}_{2}\left(\left\|s_{0}-s^{*}\right\|\right)\right)} .
$$

using Banach lemma on invertible operators. We obtain from (3), (7), (9), (12), (19), (26), (28) and (29) that

$$
\begin{aligned}
\left\|u_{0}-s^{*}\right\| & \leq\left\|t_{0}-s^{*}\right\|+\left\|\mathcal{Q}^{\prime}\left(s_{0}\right)^{-1} \mathcal{Q}^{\prime}\left(s^{*}\right)\right\| \\
& \times\left(\left\|\mathcal{Q}^{\prime}\left(s^{*}\right)^{-1}\left(\mathcal{Q}^{\prime}\left(t_{0}\right)-\mathcal{Q}^{\prime}\left(s^{*}\right)\right)\right\|+\left\|\mathcal{Q}^{\prime}\left(s^{*}\right)^{-1}\left(\mathcal{Q}^{\prime}\left(s_{0}\right)-\mathcal{Q}^{\prime}\left(s^{*}\right)\right)\right\|\right) \\
& \times\left\|\left(\mathcal{Q}^{\prime}\left(s_{0}\right)+\mathcal{Q}^{\prime}\left(t_{0}\right)\right)^{-1} \mathcal{Q}^{\prime}\left(s^{*}\right)\right\|\left\|\mathcal{Q}^{\prime}\left(s^{*}\right)^{-1} \mathcal{Q}\left(s_{0}\right)\right\| \\
& \leq \mathcal{H}_{1}\left(\left\|s_{0}-s^{*}\right\|\right)\left\|s_{0}-s^{*}\right\| \\
& +\frac{w_{0}\left(\left\|t_{0}-s^{*}\right\|^{q}+\left\|s_{0}-s^{*}\right\|^{q}\right)\left(1+w_{0}\left\|s_{0}-s^{*}\right\|^{q}\right)\left\|s_{0}-s^{*}\right\|}{\left(1-w_{0}\left\|s_{0}-s^{*}\right\|^{q}\right)\left(2\left(1-\mathcal{H}_{2}\left(\left\|s_{0}-s^{*}\right\|\right)\right)\right)} \\
& \leq \mathcal{H}_{1}\left(\left\|s_{0}-s^{*}\right\|\right)\left\|s_{0}-s^{*}\right\| \\
& +\frac{w_{0}\left(\mathcal{H}_{1}\left(\left\|s_{0}-s^{*}\right\|\right)^{q}\left\|s_{0}-s^{*}\right\|^{q}+\left\|s_{0}-s^{*}\right\|^{q}\right)\left(1+w_{0}\left\|s_{0}-s^{*}\right\|^{q}\right)\left\|s_{0}-s^{*}\right\|}{\left(1-w_{0}\left\|s_{0}-s^{*}\right\|^{q}\right)\left(2\left(1-\mathcal{H}_{2}\left(\left\|s_{0}-s^{*}\right\|\right)\right)\right)} \\
& =\left(\mathcal{H}_{1}\left(\left\|s_{0}-s^{*}\right\|\right)+\frac{w_{0}\left(\mathcal{H}_{1}\left(\left\|s_{0}-s^{*}\right\|\right)^{q}\left\|s_{0}-s^{*}\right\|^{q}+\left\|s_{0}-s^{*}\right\|^{q}\right)\left(1+w_{0}\left\|s_{0}-s^{*}\right\|^{q}\right)}{\left(1-w_{0}\left\|s_{0}-s^{*}\right\|^{q}\right)\left(2\left(1-\mathcal{H}_{2}\left(\left\|s_{0}-s^{*}\right\|\right)\right)\right)}\right)\left\|s_{0}-s^{*}\right\| \\
& =\mathcal{H}_{3}\left(\left\|s_{0}-s^{*}\right\|\right)\left\|s_{0}-s^{*}\right\|<\left\|s_{0}-s^{*}\right\|<R .
\end{aligned}
$$

Finally, we use (3), (8), (9), (13), (19), (26), (28) (29) and (30) to deduce

$$
\begin{aligned}
& \left\|s_{1}-s^{*}\right\| \leq\left\|u_{0}-s^{*}\right\|+\left\|\left(\mathcal{Q}^{\prime}\left(s_{0}\right)+\mathcal{Q}^{\prime}\left(t_{0}\right)\right)^{-1} \mathcal{Q}^{\prime}\left(s^{*}\right)\right\| \\
& \times\left(\left\|\mathcal{Q}^{\prime}\left(s^{*}\right)^{-1}\left(\mathcal{Q}^{\prime}\left(t_{0}\right)-\mathcal{Q}^{\prime}\left(s^{*}\right)\right)\right\|+\left\|\mathcal{Q}^{\prime}\left(s^{*}\right)^{-1}\left(\mathcal{Q}^{\prime}\left(s_{0}\right)-\mathcal{Q}^{\prime}\left(s^{*}\right)\right)\right\|\right. \\
& \left.+2\left\|\mathcal{Q}^{\prime}\left(s^{*}\right)^{-1}\left(\mathcal{Q}^{\prime}\left(s_{0}\right)-\mathcal{Q}^{\prime}\left(s^{*}\right)\right)\right\|+2\left\|\mathcal{Q}^{\prime}\left(s^{*}\right)^{-1} \mathcal{Q}^{\prime}\left(s^{*}\right)\right\|\right) \\
& \times\left\|\mathcal{Q}^{\prime}\left(s_{0}\right)^{-1} \mathcal{Q}^{\prime}\left(s^{*}\right)\right\|\left\|\int_{0}^{1} \mathcal{Q}^{\prime}\left(s^{*}\right)^{-1} \mathcal{Q}^{\prime}\left(s^{*}+\xi\left(u_{0}-s^{*}\right)\right)\left(u_{0}-s^{*}\right) d \xi\right\| \\
& \leq \mathcal{H}_{3}\left(\left\|s_{0}-s^{*}\right\|\right)\left\|s_{0}-s^{*}\right\| \\
& +\frac{\left[w_{0}\left(\left\|t_{0}-s^{*}\right\|^{q}+3\left\|s_{0}-s^{*}\right\|^{q}\right)+2\right]\left(1+w_{0}\left\|s_{0}-s^{*}\right\|^{q}\right)\left\|u_{0}-s^{*}\right\|}{\left(1-w_{0}\left\|s_{0}-s^{*}\right\|^{q}\right)\left(2\left(1-\mathcal{H}_{2}\left(\left\|s_{0}-s^{*}\right\|\right)\right)\right)}
\end{aligned}
$$




$$
\begin{aligned}
& \leq \mathcal{H}_{3}\left(\left\|s_{0}-s^{*}\right\|\right)\left\|s_{0}-s^{*}\right\| \\
& +\frac{\left[w_{0}\left(\mathcal{H}_{1}\left(\left\|s_{0}-s^{*}\right\|\right)^{q}\left\|s_{0}-s^{*}\right\|^{q}+3\left\|s_{0}-s^{*}\right\|^{q}\right)+2\right]\left(1+w_{0}\left\|s_{0}-s^{*}\right\|^{q}\right) \mathcal{H}_{3}\left(\left\|s_{0}-s^{*}\right\|\right)\left\|s_{0}-s^{*}\right\|}{\left(1-w_{0}\left\|s_{0}-s^{*}\right\|^{q}\right)\left(2\left(1-\mathcal{H}_{2}\left(\left\|s_{0}-s^{*}\right\|\right)\right)\right)} \\
& =\left(\mathcal{H}_{3}\left(\left\|s_{0}-s^{*}\right\|\right)\right. \\
& \left.+\frac{\left[w_{0}\left(\mathcal{H}_{1}\left(\left\|s_{0}-s^{*}\right\|\right)^{q}\left\|s_{0}-s^{*}\right\|^{q}+3\left\|s_{0}-s^{*}\right\|^{q}\right)+2\right]\left(1+w_{0}\left\|s_{0}-s^{*}\right\|^{q}\right) \mathcal{H}_{3}\left(\left\|s_{0}-s^{*}\right\|\right)}{\left(1-w_{0}\left\|s_{0}-s^{*}\right\|^{q}\right)\left(2\left(1-\mathcal{H}_{2}\left(\left\|s_{0}-s^{*}\right\|\right)\right)\right)}\right)\left\|s_{0}-s^{*}\right\| \\
& =\mathcal{H}_{4}\left(\left\|s_{0}-s^{*}\right\|\right)\left\|s_{0}-s^{*}\right\|<\left\|s_{0}-s^{*}\right\|<R .
\end{aligned}
$$

Thus, the estimate (24) is true for $k=0$. We obtain the estimates (22)-(24) by using $s_{k}, t_{k}$ and $s_{k+1}$ instead of $s_{0}, t_{0}$ and $s_{1}$ respectively in the earlier estimations. Since $\left\|s_{k+1}-s^{*}\right\| \leq \mathcal{H}_{4}(R)\left\|s_{k}-s^{*}\right\|<R$, we confirm that $s_{k+1} \in B\left(s^{*}, R\right)$ and $\lim _{k \rightarrow \infty} s_{k}=s^{*}$. The uniqueness part is proved by considering another solution $t^{*}\left(\neq s^{*}\right) \in B\left(s^{*}, \Delta\right) \cap \Omega$ of $\mathcal{Q}(s)=0$. Let $\mathcal{G}=$ $\int_{0}^{1} \mathcal{Q}^{\prime}\left(t^{*}+\xi\left(s^{*}-t^{*}\right)\right) d \xi$. From equation (15), we get

$$
\begin{aligned}
\left\|\mathcal{Q}^{\prime}\left(s^{*}\right)^{-1}\left(\mathcal{G}-\mathcal{Q}^{\prime}\left(s^{*}\right)\right)\right\| & \leq \int_{0}^{1} w_{0}\left\|t^{*}+\xi\left(s^{*}-t^{*}\right)-s^{*}\right\|^{q} d \xi \\
& \leq \frac{w_{0}}{q+1}\left\|s^{*}-t^{*}\right\|^{q} \\
& \leq \frac{w_{0} \Delta^{q}}{q+1}<1 .
\end{aligned}
$$

The invertability of $\mathcal{G}$ and the identity $0=\mathcal{Q}\left(s^{*}\right)-\mathcal{Q}\left(t^{*}\right)=\mathcal{G}\left(s^{*}-t^{*}\right)$ imply that $s^{*}=t^{*}$.

\section{Numerical examples}

We validate our theoretical results in this section. We compare the convergence radii of the method (3), obtained by our proposed analysis, with the radii obtained in the earlier study [22]. It is concluded that the proposed technique produces bigger convergence radii for the method (3) than the previous work [22].

Example 1 [22] Let $\mathcal{Q}$ is defined on $\bar{B}(0,1)$ for $\left(s_{1}, s_{2}, s_{3}\right)^{t}$ by

$$
\mathcal{Q}(s)=\left(e^{s_{1}}-1, \frac{e-1}{2} s_{2}^{2}+s_{2}, s_{3}\right)^{t}
$$

We have $s^{*}=(0,0,0)^{t}, q=1, w_{0}=e-1$ and $w_{1}=1.789572397$. The radius $R$ is obtained using the proposed analytical result (see Table 1). 
Table 1. Comparison of convergence radius for Example 1

\begin{tabular}{ccccr}
\hline & Proposed Analysis & Earlier study [22] \\
\hline$\Delta_{1}$ & $\Delta_{3}$ & $\Delta_{4}$ & $R$ & $r$ \\
0.382692 & 0.215469 & 0.114489 & 0.114489 & 0.075870 \\
\hline
\end{tabular}

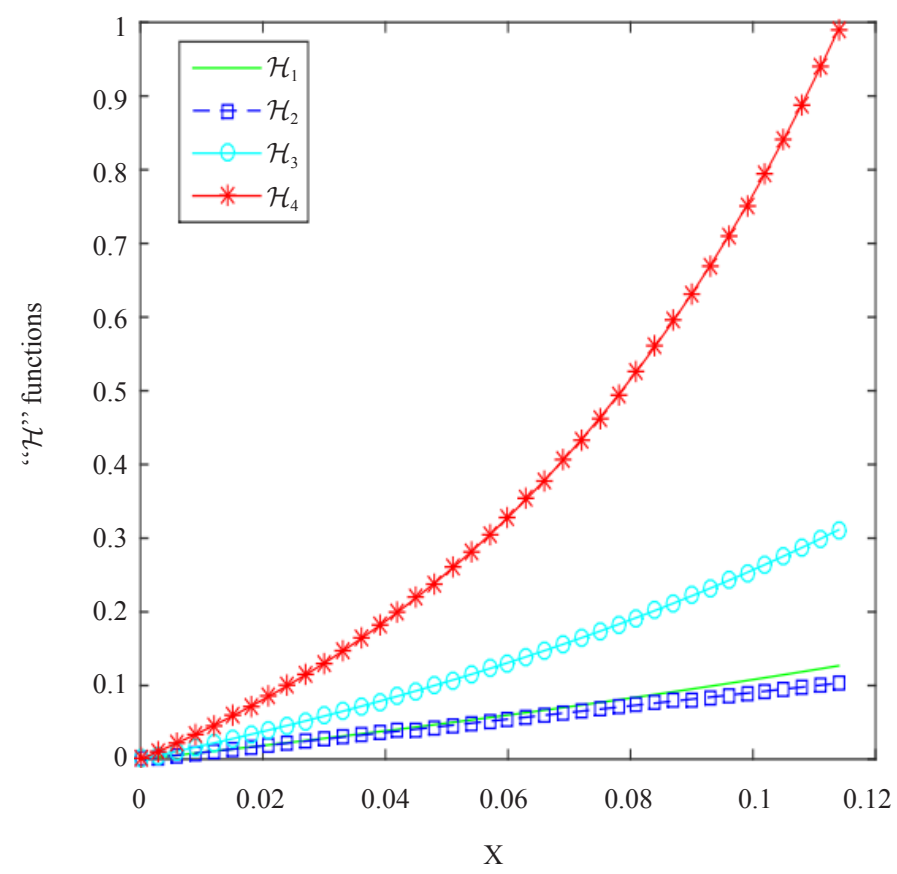

Figure 1. $\mathcal{H}$ functions for Example 1

Example 2 [22] Define $\mathcal{Q}$ on $\Omega=\left[-\frac{1}{2}, \frac{5}{2}\right]$ by

$$
\mathcal{Q}(s)=\left\{\begin{array}{ll}
s^{3} \ln \left(s^{2}\right)+s^{5}-s^{4}, & \text { if } s \neq 0 \\
0, & \text { if } s=0
\end{array} .\right.
$$

We have $s^{*}=1, q=1$ and $w_{0}=w_{1}=96.6628$. The value of $R$ is given in Table 2 .

Table 2. Comparison of convergence radius for Example 2

\begin{tabular}{ccccc}
\hline & \multicolumn{2}{c}{ Proposed Analysis } & Earlier study [22] \\
\hline$\Delta_{1}$ & $\Delta_{3}$ & $\Delta_{4}$ & $R$ & $r$ \\
0.006896 & 0.003865 & 0.002055 & 0.002055 & 0.001362 \\
\hline
\end{tabular}




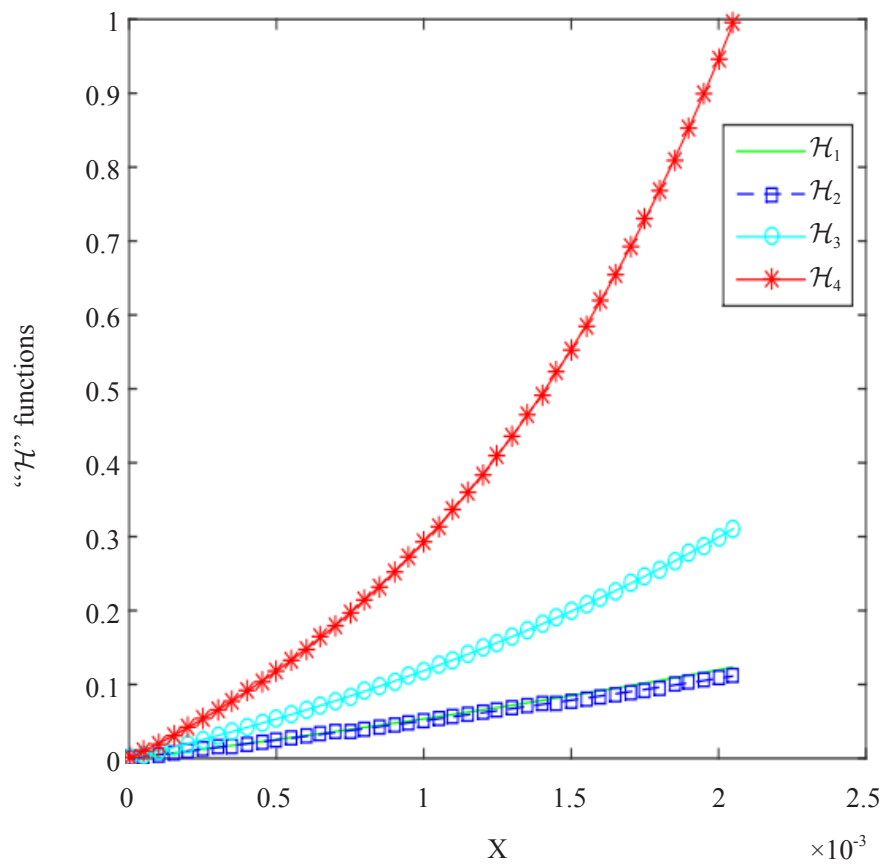

Figure 2. $\mathcal{H}$ functions for Example 2

Example 3 [22] Consider the mixed Hammerstein type integral equation given by

$$
\mathcal{Q}(s)(w)=s(w)-\int_{0}^{1} G_{1}(w, y)\left(s(y)^{\frac{5}{2}}+\frac{s(y)^{2}}{2}\right) d y
$$

where $s(w) \in C[0,1]$. We have $s^{*}=0$. Also, $q=1$ and $w_{0}=w_{1}=\frac{1}{8}\left(\frac{5}{2} \sqrt{2}+1\right)$. In Table 3 , the radius $R$ is provided.

Table 3. Comparison of convergence radius for Example 3

\begin{tabular}{ccccc}
\hline \multicolumn{3}{c}{ Proposed Analysis } & Earlier study [22] \\
\hline$\Delta_{1}$ & $\Delta_{3}$ & $\Delta_{4}$ & $R$ & $r$ \\
1.175899 & 0.658908 & 0.350378 & 0.350378 & 0.232141 \\
\hline
\end{tabular}

Example 4 [28] Consider the nonlinear integral equation given by

$$
\mathcal{Q}(s)(w)=s(w)-3 \int_{0}^{1} G_{1}(w, y) s(y)^{\frac{5}{4}} d y
$$

where $s(w) \in C[0,1]$ and $G_{1}(w, y)$ is Green's function. We have $s^{*}=0$. Also, $q=0.25$ and $w_{0}=w_{1}=\frac{15}{32}$. The value of $R$ is presented in Table 4. 
Table 4. Comparison of convergence radius for Example 4

\begin{tabular}{ccccc}
\hline & \multicolumn{2}{c}{ Proposed Analysis } & Earlier study [22] \\
\hline$\Delta_{1}$ & $\Delta_{3}$ & $\Delta_{4}$ & $R$ & $r$ \\
1.973081 & 0.060989 & 0.005851 & 0.005851 & Cannot be computed \\
\hline
\end{tabular}

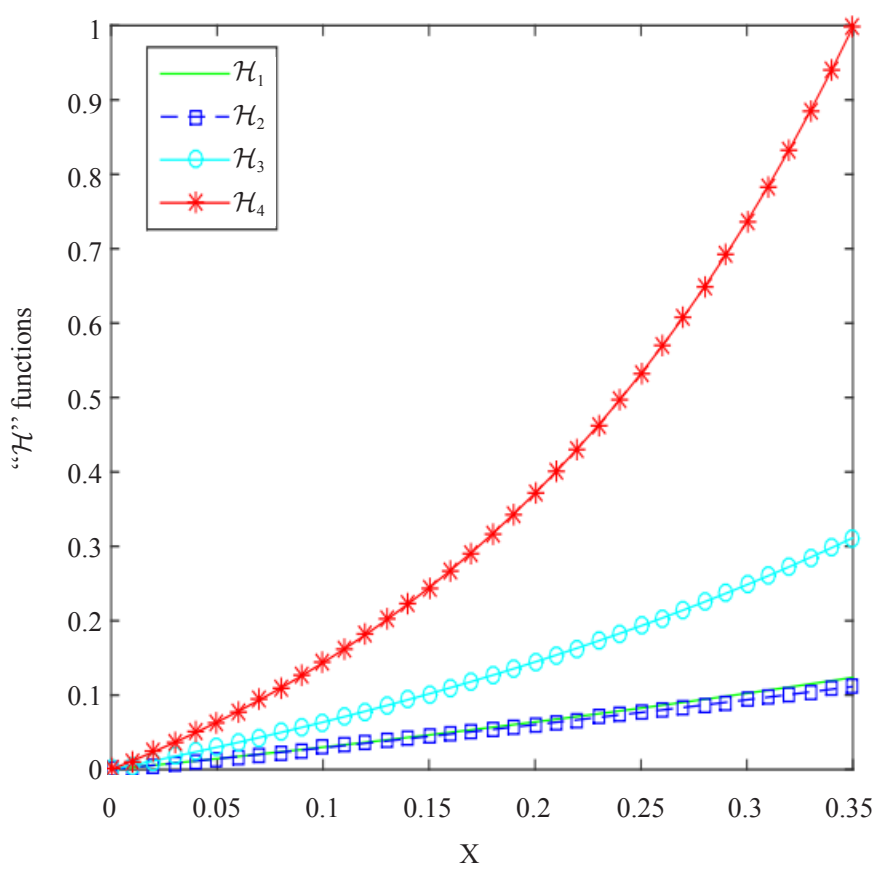

Figure 3. $\mathcal{H}$ functions for Example 3

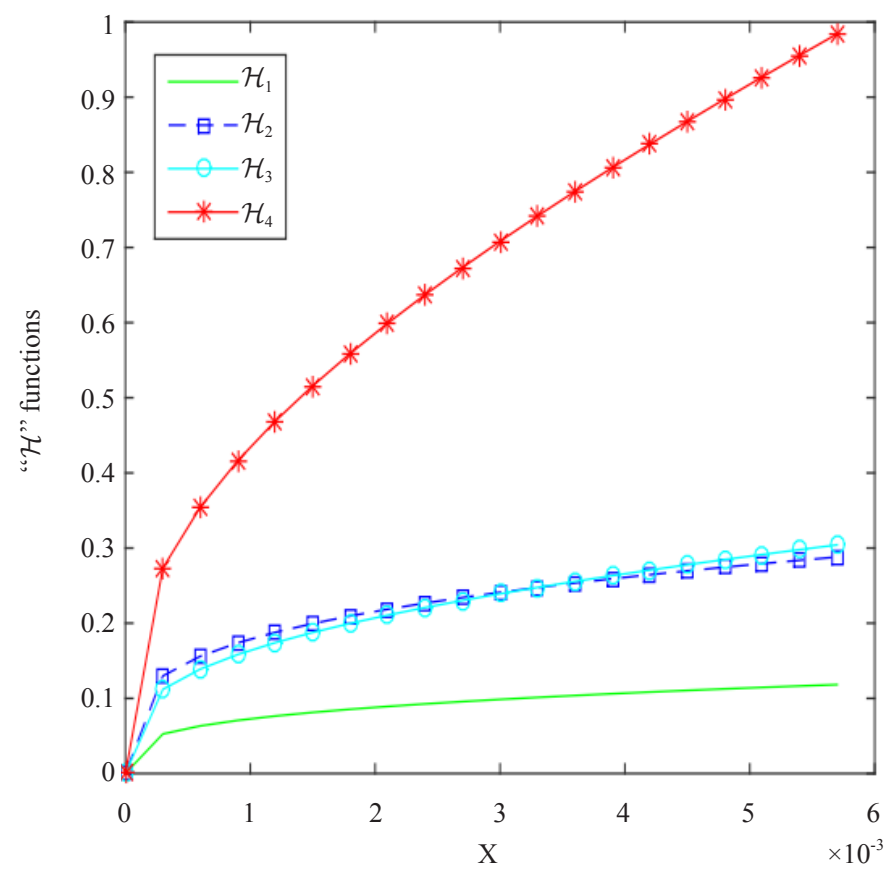

Figure 4. $\mathcal{H}$ functions for Example 4 
Thus, we show the convergence of the scheme (3) for Example 4 with radius $R=0.005851$.

\section{Conclusions}

Local convergence analysis of a fifth-order nonlinear equation solver is presented to extend its applicability and convergence domain. This study generalized the local analysis based on Lipschitz condition. It is concluded from the numerical tests that our analysis provides a larger domain of convergence, in comparison with the previous work, for the scheme (3). Also, our theoretical conclusions worked well in the situation where the earlier analysis based on Lipschitz constants cannot be used.

\section{References}

[1] Argyros IK. Convergence and Application of Newton-type Iterations. Berlin: Springer; 2008.

[2] Argyros IK, Cho YJ, Hilout S. Numerical Methods for Equations and its Applications. New York: CRC Press; 2012.

[3] Ortega JM, Rheinboldt WC. Iterative Solution of Nonlinear Equations in Several Variables. New York: Academic Press; 1970.

[4] Petković MS, Neta B, Petković L, Dz̃unić D. Multipoint Methods for Solving Nonlinear Equations. Amsterdam: Elsevier; 2013.

[5] Rall LB. Computational Solution of Nonlinear Operator Equations. New York: Robert E. Krieger; 1979.

[6] Traub JF. Iterative Methods for Solution of Equations. Englewood Cliffs: Prentice-Hal; 1964.

[7] Behl R, Cordero A, Motsa SS, Torregrosa JR. Construction of fourth-order optimal families of iterative methods and their dynamics. Applied Mathematics and Computation. 2015; 271: 89-101.

[8] Chun C, Lee MY, Neta B, Džunić J. On optimal fourth-order iterative methods free from second derivative and their dynamics. Applied Mathematics and Computation. 2012; 218(11): 6427-6438.

[9] Cordero A, Torregrosa JR. Variants of Newton's method for functions of several variables. Applied Mathematics and Computation. 2007; 183: 199-208.

[10] Darvishi MT, Barati A. A fourth-order method from quadrature formulae to solve systems of nonlinear equations. Applied Mathematics and Computation. 2007; 188: 257-261.

[11] Frontini M, Sormani E. Some variant of Newton's method with third order convergence. Applied Mathematics and Computation. 2003; 140: 419-426.

[12] Grau-Sánchez M, Grau Á, Noguera M. On the computational efficiency index and some iterative methods for solving systems of nonlinear equations. Journal of Computational and Applied Mathematics. 2011; 236: 12591266.

[13] Homeier HHH. A modified Newton method with cubic convergence: the multivariable case. Journal of Computational and Applied Mathematics. 2004; 169: 161-169.

[14] Kou J, Li Y, Wang X. A composite fourth-order iterative method for solving non-linear equations. Applied Mathematics and Computation. 2007; 184: 471-475.

[15] Noor MA, Wassem M. Some iterative methods for solving a system of nonlinear equations. Applied Mathematics and Computation. 2009; 57: 101-106.

[16] Özban AY. Some new variants of Newton's method. Applied Mathematics Letters. 2004; 17: 677-682.

[17] Sharma D, Parhi SK, Sunanda SK. A new class of fifth and sixth order root-finding methods with its dynamics and applications. Contemporary Mathematics. 2020; 1(5): 429-444.

[18] Weerakoon S, Fernando TGI. A variant of Newton's method with accelerated third-order convergence. Applied Mathematics Letters. 2000; 13: 87-93.

[19] Argyros IK, Magreñán ÁA. A study on the local convergence and the dynamics of Chebyshev-Halley-type methods free from second derivative. Numerical Algorithms. 2015; 71(1): 1-23.

[20] Argyros IK, George S, Magreñán ÁA. Local convergence for multi-point-parametric Chebyshev-Halley-type methods of higher convergence order. Journal of Computational and Applied Mathematics. 2015; 282: $215-224$.

[21] Sharma D, Parhi SK, Sunanda SK. Extending the convergence domain of deformed Halley method under $\omega$ condition in Banach spaces. Boletin de la Sociedad Matematica Mexicana. 2021; 27(2): 32. Available from: doi: 
10.1007/s40590-021-00318-2.

[22] Argyros IK, George S. Local convergence of a fifth convergence order method in Banach space. Arab Journal of Mathematical Sciences. 2017; 23: 205-214.

[23] Cordero A, Ezquerro JA, Hernandez-Veron MA. On the local convergence of a fifth-order iterative method in Banach spaces. Applied Mathematics and Computation. 2015; 251: 396-403.

[24] Maroju P, Magreñán ÁA, Sarría Í, Kumar A. Local convergence of fourth and fifth order parametric family of iterative methods in Banach spaces. Journal of Mathematical Chemistry. 2020; 58: 686-705.

[25] Martínez E, Singh S, Hueso JL, Gupta DK. Enlarging the convergence domain in local convergence studies for iterative methods in Banach spaces. Applied Mathematics and Computation. 2016; 281: 252-265.

[26] Sharma JR, Argyros IK. Local convergence of a Newton-Traub composition in Banach spaces. SeMA Journal. 2017; 75(1): 57-68.

[27] Singh S, Gupta DK, Badoni RP, Martínez E, Hueso JL. Local convergence of a parameter based iteration with Hölder continuous derivative in Banach spaces. Calcolo. 2017; 54(2): 527-539.

[28] Amat S, Argyros IK, Busquier S, Hernández-Verón MA, Martínez E. On the local convergence study for an efficient k-step iterative method. Journal of Computational and Applied Mathematics. 2018; 343: 753-761.

[29] Argyros IK, Sharma, D, Parhi, SK, Sunanda SK. On the convergence, dynamics and applications of a new class of nonlinear system solvers. International Journal of Applied and Computational Mathematics. $2020 ; 6(5): 142$. Available from: doi: 10.1007/s40819-020-00893-4.

[30] Argyros IK, Cho YJ, George S. Extending the convexity of nonlinear image of a ball appearing in optimization. Contemporary Mathematics. 2020; 1(4): 209-214. Available from: doi: 10.37256/cm.142020405.

[31] Argyros IK, George S. High convergence order Q-step methods for solving equations and systems of equations. Contemporary Mathematics. 2020; 1(3); 119-126. Available from: doi: 10.37256/cm.132020403.

[32] Argyros IK, Regmi S. Undergraduate Research at Cameron University on Iterative Procedures in Banach and Other Spaces. New York: Nova Science Publisher; 2019.

[33] Ezquerro JA, González D, Hernández MA. On the local convergence of Newton's method under generalized conditions of Kantorovich. Applied Mathematics Letters. 2013; 26(5): 566-570.

[34] Hernández MA, Rubio MJ. On the local convergence of a Newton-Kurchatov-type method for non-differentiable operators. Applied Mathematics and Computation. 2017; 304: 1-9.

[35] Regmi S. Optimized Iterative Methods with Applications in Diverse Disciplines. New York: Nova Science Publisher; 2021. 